\title{
Philosophie für Ärzte und Therapeuten - ein Erfahrungsbericht
}

\author{
Vor ungefähr drei Jahren berichtete die Schweizerische Ärztezeitung über den \\ Lehrgang «Philosophie für Fachleute aus Medizin und Psychotherapie», der \\ damals neu im Angebot der medizinischen Weiterbildung an der Universität \\ Zürich war. Unterdessen haben über hundert Berufsleute den Lehrgang \\ besucht. Eine Psychoanalytikerin und ein Arzt, die beide seit mehreren Seme- \\ stern dabei sind, erzählen, was sie von diesem Angebot halten und was ihnen \\ die philosophische Auseinandersetzung bringt.
}

Korrespondenz: Lisa Heller

Gasometerstrasse 7 CH-8005 Zürich

Tel. 0442731532

liheller@swissonline.ch
«Es tut wahnsinnig gut, sich von theoretischen Gebäuden zu lösen, in denen man sich sehr gut auskennt, plötzlich dieses Heimatgefühl zu verlieren. Man muss neu zu denken beginnen», sagt die Psychoanalytikerin Susanne Fernandez.

«Der Kurs heisst Philosophie, aber [...] eigentlich ist es Ideengeschichte. Man setzt sich mit Ideen auseinander, die man vielleicht selber auch schon gehabt hat - selbst bei einem Text von de Sade, bei dem man Abscheu empfindet. Dann merkt man, dass dieser unglaublich gescheit formuliert und begründet ist, dass all die Grausamkeiten nicht so dumm sind. Da wird man unsicher und mit eigenen Vorstellungen konfrontiert», erzählt der Arzt Jean Lucien L'Eplattenier.

Fernandez und L'Eplattenier beziehen sich in ihren Aussagen auf den Lehrgang «Philosophie für Fachleute aus Medizin und Psychotherapie», der seit rund vier Jahren im Rahmen der Weiterbildung an der Universität Zürich angeboten wird. Beide haben sich vor sieben beziehungsweise fünf Semestern zur Teilnahme daran entschlossen, weil sie ihr Interesse für Philosophie vertiefen wollten. Beide arbeiten seit Jahren in ihren Berufen und sind insofern typische Lehrgangteilnehmer. «Die Menschen, die zu uns kommen, haben alle langjährige Praxiserfah-

\section{Philosophie pour médecins et psychothérapeutes}

II y a trois ans, le Bulletin des médecins suisses a rendu compte d'un cours de formation continue intitulé «Philosophie pour les professionnels du corps médical et psychothérapeutique» qui, à l'époque, était une nouvelle offre de l'Université de Zurich. La mise en place du cours était motivée par le fait que ces professionnels avaient et ont le sentiment que leur formation ainsi que leurs méthodes ne leur permettent pas d'aborder les questions essentielles de leur pratique professionnelle au quotidien. II fallait donc revisiter la tradition philosophique de ces disciplines afin de s'ouvrir à de nouvelles solutions aux problèmes rencontrés.

Le cours de base traite de la manière philosophique de penser, questionner et raisonner, tandis que les séminaires s'appliquent à approfondir des sujets tels que la peur, la honte ou la liberté.

Entre-temps plus de cent participants ont assisté ou assistent à la formation dont un médecin trouve que le cours est un complément bienvenu aux autres formations continues, qu'il est adapté à la profession des médecins et qu'il leur tend un miroir instructif. Par ailleurs, une psychanalyste dit que c'est un bonheur de se libérer des théories qu'on connaît, certes, très bien et qu'il faut renouveler sa pensée.

Pour plus d'informations (en allemand):

www.philopsycho.ch 
rung», sagt der Philosoph und Psychoanalytiker Daniel Strassberg, der den Lehrgang leitet. «Offenbar macht sich nach einer gewissen Zeit das Bedürfnis bemerkbar, einen Schritt über die fachspezifische Weiterbildung hinauszugehen. Aber natürlich würden wir uns auch über jüngere Teilnehmer freuen.»

Strassberg hat den Lehrgang zusammen mit dem emeritierten Philosophieprofessor Helmut Holzhey und dem Direktor der Psychiatrischen Universitätsklinik Zürich, Daniel Hell, initiiert. «Viele Mediziner, Psychiaterinnen und Psychotherapeuten leiden daran, dass sie weder die psychotherapeutische Ausbildung noch die biologisch-pharmakologische Vorgehensweise dazu befähigt, einen Umgang mit grundsätzlichen Fragen $\mathrm{zu}$ finden, denen sie in ihrem Beruf zwangsläufig ausgesetzt sind», beschreiben die drei Kursleiter die Ausgangslage, die sie zu ihrem Schritt bewogen hat. "Auch die Fähigkeit zur Reflexion über das eigene Handeln, die in der Selbsterfahrung erworben wird, stösst da an Grenzen, wo grundsätzliche Fragen des Lebens und des Sinns berührt werden. Die Folgen davon sind oft genug Burn-out oder theoretischer Dogmatismus.»

Hier nun wollen sie mit ihrem Lehrgang ansetzen. Durch den Rückgriff auf Ideen, Vorstellungen und Konzepte der Philosophie soll Ärzten und Therapeuten ein anderer Zugang zu ihren Erfahrungen und Schwierigkeiten im Berufsalltag vermittelt werden. Die Philosophie biete sich deshalb dafür an, weil Medizin und Psychologie als Einsichten über das Wesen des Menschen und der Natur und als Heilung der Seele jahrhundertelang eng mit dieser verflochten gewesen seien. «Erst mit der Etablierung von Medizin und Psychologie als eigenständige wissenschaftliche Fachgebiete und dem damit verbundenen Vormarsch des rein naturwissenschaftlichen Denkens wurde diese Tradition gekappt, wobei ein reicher Fundus an Wissen verlorenging.»

Als Einstieg führt jeweils im Sommersemester ein Grundkurs anhand von klassischen Texten in die spezifische Weise des philosophischen Denkens, Fragens und Argumentierens ein. «Man lernt, wie man an einen philosophischen Text herangeht, ihn einordnet, Begriffe klärt und die Position eines Denkers herausarbeitet», erklärt Fernandez. «Zum Beispiel schaut man, was man ergänzen könnte, was aus dem eigenen Erleben fehlt. Oder man sucht eine Gegenposition, um einem spezifischen Denken auf den Grund zu gehen.»

In weiterführenden Lektüre- und Überblickseminaren wird dann in Form von gemeinsamen
Lektüren oder von Vorlesungen und Gesprächen jeweils ein Semester lang ein Thema behandelt, das an der beruflichen Praxis orientiert ist, zum Beispiel Angst, Scham, Freiheit oder Liebe und Freundschaft. Wie sich das Gespräch entwickelt, ist offen. Es gebe sehr gute und dann wieder eher schleppende Sitzungen, sagt Strassberg, insgesamt stellt er aber ein steigendes Niveau fest. «Diejenigen, die schon jahrelang dabei sind, kommen langsam in die Art des Denkens rein.»

Für Fernandez hat sich die Hoffnung bestätigt, dass durch die Nähe der behandelten Themen zu ihrer täglichen Arbeit in der Therapie neue Horizonte aufgehen. Ihr Denken habe sich durch diese Auseinandersetzung geschärft, sei mehrdimensionaler geworden, sagt sie und kommt auf das Thema Angst zu sprechen: «Die Möglichkeit, philosophische mit analytischen oder psychologischen Fragestellungen zu konfrontieren, ergibt interessante Dissonanzen. Wenn man etwa das Thema Angst philosophisch betrachtet, tut sich eine existentielle Dimension auf, die in den Angsttheorien von Freud nicht vorhanden ist.» L'Eplattenier wiederum hilft die Auseinandersetzung mit verschiedenen Denkern, Distanz zur eigenen beruflichen Praxis zu gewinnen und Positionen zu hinterfragen: «Erkenntnistheorie ist heute sehr eingeengt, fast nur noch Wissenschaftstheorie, und wir Mediziner meinen immer, dass wir den Gipfel des Wissenschaftlichen erreicht haben. Aber die Wissenschaft ist historisch gewachsen. Im Kurs erhält man Distanz und sieht, dass das, was wir tun, Teil einer Entwicklung ist.» Positiv findet er das Undogmatische und Sachliche an der Art, wie Philosophie im Lehrgang vermittelt wird. Nicht um tiefsinnige Deutungen der heutigen Medizin gehe es da, sondern um Ideen und Fragen, die jeden Menschen beschäftigen und mit denen Ärzte im Umgang mit ihren Patienten konfrontiert seien. Er bezeichnet den Lehrgang denn auch als gute spezifische Ergänzung zur Fortbildung und würde sich eine teilweise Anerkennung durch die ärztlichen Gesellschaften wünschen. «Es ist nicht irgendeine Philosophievorlesung, sondern ein Lehrgang, der auf uns ausgerichtet ist, in dem Mediziner mitmachen, man seine Position mitbringen kann und einem der Spiegel vorgehalten wird. Nicht dass man am nächsten Tag die Sprechstunde anders halten würde, aber es stillt das Bedürfnis nachzudenken.»

Angesprochen ist damit die Frage, wie der Lehrgang Ärzten und Therapeuten in ihrem Beruf konkret zugute kommt. Der Lehrgang ziele nicht in erster Linie auf die Vermittlung philosophischen Wissens oder gar auf eine neue Theo- 
riebildung ab, vielmehr soll er für eine andere Art des Denkens öffnen, schreiben die Kursleiter dazu. Dies schlage sich zwar nicht unmittelbar in einer besseren oder effizienteren therapeutischen Praxis und vielleicht nicht einmal in einem besseren Verständnis der Patienten nieder. «Aber mittelbar versprechen die Öffnung der alltäglichen Routine und die Erweiterung des Denkhorizontes eine Belebung des Berufsalltags und eine Vertiefung der darin gemachten Erfahrungen, die schliesslich auch den Patientinnen und Patienten zugute kommt.»

Fernandez ihrerseits beschreibt die Vermittlung zwischen dem eigenen Spezialgebiet und der Philosophie als gefühlsmässigen und intellektuellen Prozess, der dem einzelnen nicht mundgerecht geboten werden könne, sondern den jeder selber leisten müsse. Die Frage der Umsetzung stehe immer mal wieder im Raum, fügt sie an, so dass man in einzelnen Sitzungen das Bedürfnis nach einer Anleitung herausspüren könne, wie das dort Erarbeitete nun anzuwenden wäre. «Aber die Auseinandersetzung muss erst wirken und nachhallen, bevor sie dann plötzlich in die therapeutische Praxis reinkommt.»

Susanne Fernandez und Jean Lucien L'Eplattenier haben verschiedene Berufe und wissenschaftliche Hintergründe. Dass sich der Lehrgang sowohl an nichtärztliche Therapeuten als auch an Psychiaterinnen und Mediziner richtet, empfinden beide als Bereicherung. «Der Doppelblick ist wichtig», sagt L'Eplattenier und kommt insbesondere auf die kommunikative Kompetenz zu sprechen. Er fühle da ein gewisses Manko bei sich, ein Psychiater habe einen ganz anderen Zugang zum Wort. Fernandez indessen merkt an, dass sie von Kollegen und Kolleginnen, die viel über Physiologie, das Innere des Körpers oder Neurologie wüssten, immer wieder etwas dazulernen könne.

Weitere Informationen und Anmeldung: www.philopsycho.ch

Anmeldeschluss für den nächsten Grundkurs: 7. März 2008 